\title{
Dynastic inequality, mobility and equality of opportunity
}

\author{
Ravi Kanbur' ${ }^{1}$ Joseph E. Stiglitz ${ }^{2}$
}

Received: 7 March 2016 / Accepted: 10 March 2016 / Published online: 13 July 2016

(C) Springer Science+Business Media New York 2016

\begin{abstract}
One often heard counter to the concern about rising income and wealth inequality is that it is wrong to focus on inequality of outcomes in a "snapshot". Intergenerational mobility and "equality of opportunity", so the argument goes, is what matters for normative evaluation. In response to this counter, we ask what pattern of intergenerational mobility leads to lower inequality not between individuals but between the dynasties to which they belong? And how does this pattern in turn relate to commonly held views on what constitutes equality of opportunity? We revive and revisit here our earlier contributions which were in the form of working papers (Kanbur and Stiglitz 1982, 1986) in order to engage with the current debate. Focusing on bistochastic transition matrices in order to hold constant the steady state snapshot income distribution, we develop an explicit partial ordering which ranks matrices on the criterion of inequality between infinitely lived dynasties. A general interpretation of our result is that when comparing two transition matrices, if one matrix is "further away" from the identity matrix then it will lead to lower dynastic inequality. More specifically, the result presents a computational procedure to check if one matrix dominates another on dynastic inequality. We can also assess "equality of opportunity", defined as identical prospects irrespective of starting position. We find that this is not necessarily the mobility pattern which minimizes dynastic inequality.
\end{abstract}

Keywords Dynastic inequality · Mobility · Equality of opportunity

Ravi Kanbur

sk145@cornell.edu

Joseph E. Stiglitz

jes322@columbia.edu

1 Cornell University, Ithaca, NY 14850, USA

2 Columbia University, New York, NY 10027, USA 


\section{Introduction}

It is clear that global economic forces are now aligned to produce rising income and wealth inequality. There is, of course, disagreement about the relative importance of the potential drivers of these changes. The direction of technical change appears to be favoring capital over labor and, within labor, skilled labor over unskilled labor. Globalization of trade and investment is transmitting these forces to all countries, and may itself be a contributor to growing inequality. Across a wide spectrum of countries, there has been an increase in market power at least in certain sectors, and arguably, more broadly in rent seeking. ${ }^{1}$ In Latin America these underlying forces have been countered by pro-active policy. But elsewhere, including in Asia, Europe and the US, policy has not countered, and perhaps has even exacerbated, the forces for rising inequality. Not surprisingly, rising inequality is now of great concern to policy makers and the general public the world over. The phenomenal response to the book on inequality by Thomas Piketty (2014) is but one illustration of these concerns.

One often heard counter to the concern on rising inequality is that it is wrong to focus on inequality of outcome in a "snapshot". What is important is how income or wealth evolves over time, especially across generations. ${ }^{2}$ Intergenerational mobility, so the argument goes, is what matters for normative evaluation. These arguments have resurfaced in the postPiketty debates, but have actually been ever present in the discourse on inequality and public policy. Thus Friedman (1962) writes as follows in Capitalism and Freedom:

Consider two societies that have the same annual distribution of income. In one there is great mobility and change so that the positions of particular families in the income hierarchy varies widely from year to year. In the other there is great rigidity so that each family stays in the same position year after year. The one kind of inequality is a sign of dynamic change, social mobility, equality of opportunity; the other, of a status society. The confusion between the two kinds of inequality is particularly important precisely because competitive free enterprise capitalism tends to substitute the one for the other.

Friedman (1962) uses the phrase "equality of opportunity" which has also received a great deal of attention in recent years. Roemer (1998), in particular, has attempted to formalize the concept by distinguishing between two types of factors to which snapshot inequality might be attributed - circumstances and effort. Circumstances are those factors which are outside the individual's control and effort is the term for those factors for which society holds the individual responsible. A move towards equality of opportunity would reduce the influence of circumstances on outcome.

\footnotetext{
${ }^{1}$ See Stiglitz (2013).

${ }^{2}$ Some have argued that inequalities in life time incomes is much less than inequalities observed at any moment of time: in any particular year, some individuals may have a high income, others a low income, but these average out over time. Arguing that consumption is related to permanent income, some, looking at the variability of consumption, suggest that the variability of permanent income must be much lower than the variability in outcomes in any one year. Data before the crisis for the US contaminates the analysis, since a large fraction of Americans in the bottom $80 \%$ seemingly believed that they had a much higher permanent income than they did. In any case, this theoretical paper can be made to apply to either context, with an important distinction made below.
} 
Whether one can separate out circumstances from effort, empirically or even perhaps conceptually, is a live debate (see Arneson (2014), Kanbur and Wagstaff (2016)). ${ }^{3}$ But in an intergenerational context Roemer's (1998) concept has had many precursors. Thus Nancy Stokey (1998) writes as follows:

But we care about the sources of inequality as well as its extent, which is why we distinguish between equal opportunity and equal outcomes. To what extent is the claim that our society provides equal opportunity justified? How can we tell?....I am going to take the position that if economic success is largely unpredictable on the basis of observed aspects of family background, than we can reasonably claim that society provides equal opportunity. There still might be significant inequality in income across individuals, due to differences in ability, hard work, luck, and so on, but I will call these unequal outcomes.

On the other hand, if economic success is highly predictable on the basis of family background, then I think it is difficult to accept the claim that our society provides equal opportunity. In this case accidents of birth- unequal opportunity-are primary determinants of economic status.... Consequently, on a first pass we can judge whether there is equal opportunity by looking at parents and their children to see whether the economic success of the children is determined in large part by the success of their parents.

Whether current inequality in turn influences intergenerational mobility is an important question, as discussed in the recent review by Corak (2013), and by Roemer in applying his distinction between circumstances and effort to the intergenerational context (Roemer, 2004). ${ }^{4}$ But the other side of the causal link is equally important: whether less mobility leads to more inequality in the next generation. There could be an adverse feedback process, where more inequality today leads to less mobility, and that in turn leads to still more inequality in the subsequent generation, and so forth. There is now considerable evidence of the existence of a correlation between a simple measure of mobility (intergenerational correlations) and a simple measure of inequality of outcomes (Gini coefficient); and there is some literature arguing that there is evidence for both parts of the causal structure.

This paper, by contrast, focuses largely on normative issues, but underneath these normative issues are complicated relationships between mobility and observed outcomes. We wish to know whether, for instance, societies with more mobility necessarily have a higher level of social welfare, using the economists' standard welfare framework. ${ }^{5}$ Within such a normative framework, Stokey (1998) and Friedman (1962) seem to suggest that societies with more mobility have higher levels of social welfare, regardless of the degree of inequality in outcomes observed at any moment of time. To evaluate such claims, one needs to have a more general understanding of both what we mean by more mobility and an explicit normative framework. Well before Stokey (1998), Atkinson (1981a, b) had characterized

\footnotetext{
${ }^{3}$ However, both at the theoretical and at the empirical level, both in normative and descriptive models, there is much discussion of the links between inequality of outcomes and inequality of opportunity. While the theoretical work describes mobility in terms of transition matrices (the likelihood of moving from one income level to another), empirical work focuses on correlations, typically simple correlations between the income of a child and the income of the parent(s).

${ }^{4}$ There is now a growing literature in this vein; see Aaberge, Mogstad and Peragine (2011).

${ }^{5}$ Of course, many would claim that the economists' standard framework, generalizations of utilitarianism, is badly flawed. We care about fairness, and fairness is defined by equality of opportunity. In this view, equality of opportunity is a value in its own right. We will not have anything more to say on this alternative framework here.
} 
equality of opportunity in terms an intergenerational transition probability matrix with identical rows, and Shorrocks (1978) had referred to this zero correlation between parents' income and children's prospects as "perfect mobility". Atkinson and Shorrocks, and others, had also noted an alternative type of transition matrix which might capture the notion of high mobility, namely one with strong negative correlation between parents' income and children's expected prospects, and Shorrocks (1978) noted that these two views - "equality of opportunity" and "negative correlation" - were not necessarily consistent with each other.

We can of course make a direct set of arguments favoring one of these views, and some of the literature does this. ${ }^{6}$ But much of the literature relates views on intergenerational mobility to specific social welfare functions, on the grounds that this allows precision on the nature of the value judgments in play. Starting from the early work of Atkinson (1981a, b), and Atkinson and Bourguignon (1982) there is now a very large literature in this vein. Our earlier working papers, Kanbur and Stiglitz $(1982,1986)$ were in this strand of literature, with a particular focus on dynastic inequality. Since those papers, the literature has seen tremendous growth. ${ }^{7}$ However, the particular result and characterization we presented then has not been covered in this literature, and we are reviving our earlier work in this paper to engage with the current debate on mobility as a normative counter to inequality. In particular, we wish to ask, what pattern of intergenerational mobility leads to lower inequality not between individuals but between the dynasties to which they belong? And how does this pattern in turn relate to commonly held views on what constitutes equality of opportunity?

The plan of the paper is as follows. Section 2 introduces the notation of intergenerational transition matrices, and reviews the basic literature to set up the analytical problem we are addressing. Section 3 presents a characterization result which allows a partial ordering on intergenerational mobility matrices with respect to dynastic inequality. Section 4 interprets and discusses the result through particular examples. Section 5 concludes with an agenda for future research.

\section{The model and a standard result}

Let us specify the $n x n$ transition matrix:

$$
A=\left\|a_{i j}\right\| ; a_{i j} \geq 0, \sum_{j=1}^{n} a_{i j}=1
$$

whose typical element $a_{i j}$ is the probability of movement from income category $i$ to income category $j$. In our case the transition can best be thought of as inter-generational. ${ }^{8}$ Let there be $n$ categories of incomes with income levels

$$
y_{1} \leq y_{2} \leq y_{3} \leq \ldots \leq y_{n}
$$

\footnotetext{
${ }^{6}$ Thus "equality of opportunity" type characterizations have been appealed to directly by Roemer (1998) and by the earlier literature of which Dworkin (1981) is a prime example.

${ }^{7}$ Comprehensive surveys of the earlier literature include the one by Fields and Ok (1999). Examples of papers in this vein include Markandya (1984), Chakravarty, Dutta and Weymark (1985) and Dardanoni (1993). More recent references are given in Corak (2013) and in Amiel et al. (2013).

${ }^{8}$ If the incomes are those of an individual in a particular year, and there were perfect capital markets, all that would matter is the present discounted value of income over his life, i.e. if individuals lived for two periods and the interest rate were zero, it is the inequality in $y_{t}+y_{t+1}$ that we would study. This is a very special case of the more general model that we formulate below, with a linear utility function. Implicitly, in our more general formulation, we do not allow intertemporal smoothing.
} 
For simplicity, the transition matrix $A$ will be assumed to be constant over time and, where appropriate, the probability that a particular income category is occupied will be identified with the proportion of people who actually end up in that category.

An oft-encountered view on what constitutes greater mobility may be characterized as the "diagonals view". Thus a transition matrix $A=\left\|a_{i j}\right\|$ is said to exhibit greater mobility than a transition matrix $B=\left\|b_{i j}\right\|$ if $a_{i i} \leq b_{i i}$ for all $\mathrm{i}$ with strict inequality for some $\mathrm{i}$, in other words each diagonal element of $A$ is no larger than the corresponding diagonal element of $B$. A "strong diagonals view" can be characterized as saying that $a_{i j} \geq b_{i j}$ for all $i \neq j$ with strict inequality for some $i \neq j$. Shorrocks (1978) called this property of a mobility matrix "monotonicity".

The extreme of immobility in the "diagonals view" is of course the case of the Identity matrix:

$$
A=I ; a_{i j}=\delta_{i j}=\left\{\begin{array}{l}
1 \text { for } i=j \\
0 \text { for } i \neq j
\end{array}\right.
$$

In this case, families' status remains unchanged generation after generation. Strong monotonicity simply means that there is a smaller probability of remaining in the same position, and a higher probability of going into every other position.

The other extreme is sometimes argued to be the matrix which has ones along the nonleading diagonal and zeros elsewhere:

$$
A=K ; a_{i j}=k_{i j}=\left\{\begin{array}{l}
1 \text { for } j=n+1-i \\
0 \text { for } j \neq n+1-i
\end{array}\right.
$$

Atkinson (1981a) calls this the case of "complete reversal". Those at the bottom move to the top and vice versa. Atkinson contrasts this with another view of perfect mobility, namely that of "equality of opportunity" as indicated by independence of future prospects from the current situation. This is given by a matrix whose rows are identical

$$
E=\left[\begin{array}{c}
a^{\prime} \\
\sim \\
a^{\prime} \\
a^{\prime} \\
\sim
\end{array}\right]
$$

where $a^{\prime}$ represents the vector of transition probabilities.

Shorrocks (1978) also uses (5) to define his property PM ("perfect mobility"). As Shorrocks goes on to show, one cannot both hold the "diagonals view" and the "equality of opportunity view" simultaneously. For example, with

$$
A=\left[\begin{array}{ll}
0 & 1 \\
1 & 0
\end{array}\right] \text { and } B=\left[\begin{array}{ll}
1 / 2 & 1 / 2 \\
1 / 2 & 1 / 2
\end{array}\right]
$$

it should be clear that $A$ is better than $B$ on the diagonals view while the reverse is true on the equality of opportunity view. This disjuncture came to be well appreciated in the technical literature which developed since the work of Shorrocks and Atkinson (see for example the survey by Fields and Ok (1999)), and yet one finds both views present in the discourse on intergenerational mobility.

There has been considerable development in the literature through specification of explicit dynastic social welfare functions. Consider a two period world with dynasty $i$ currently having income $y_{i}$. Thus income in the next period is $y_{j}$ with probability $a_{i j}$, from 
(1). If $W\left(y_{i}, y_{j}\right)$ represents the welfare from incomes $\left(y_{i}, y_{j}\right)$, then the expected welfare of dynasty $i$ is 9

$$
V_{i}=\sum_{j=1}^{n} a_{i j} W\left(y_{i}, y_{j}\right)
$$

If $p_{i}^{o}$ is the proportion of total units in category $i$, then social welfare will be some function of the $V_{i}$ s and the $p_{i}^{o}$ s. If we suppose that social welfare $S$ is additive in dynastic welfares, then

$$
S=\sum_{i=1}^{n} p_{i}^{o} \sum_{j=1}^{n} a_{i j} W\left(\mathrm{y}_{i}, \mathrm{y}_{j}\right)
$$

This is, in fact, the social welfare function considered by Atkinson (1981a, b). Let us now impose the condition that income transitions are in a steady state, so that the snapshot distribution of income remains unchanged over time. ${ }^{10}$ Then

$$
p^{0}=p^{1}=p^{*}
$$

The class of bistochastic transition matrices i.e. those for which

$$
a_{i j} \geq 0 ; \quad \sum_{j=1}^{n} a_{i j}=\sum_{i=1}^{n} a_{i j}=1
$$

all have a steady state distribution of the form

$$
p^{*}=\left(\frac{1}{n}, \frac{1}{n}, \frac{1}{n}, \ldots, \frac{1}{n}\right)
$$

i.e. equal numbers in each category. The bistochastic assumption is made by Atkinson (1981a, b) and the subsequent literature to focus on the pure consequences of mobility, independent of the impact on the long run snapshot distribution of income. ${ }^{11}$

Focusing then just on mobility, and by analogy with multivariate stochastic dominance, a condition is derived by Atkinson (1981a, b) for one transition matrix to give a higher social welfare than another for a class of social welfare functions. If

$$
A=\left\|a_{i j}\right\| \text { and } B=\left\|b_{i j}\right\|
$$

are the two transition matrices being compared and social welfare is given by (8) with $p_{i}^{o}=p_{i}^{*}=\frac{1}{n}$ for all $\mathrm{i}$, then it can be shown that

$$
S^{A} \geq S^{B} \text { for all } W(\cdot, \cdot) \text { such that } \frac{\partial^{2} W}{\partial y_{i} \partial y_{j}} \leq 0
$$

if and only if

$$
M=\sum_{i=1}^{k} \sum_{j=1}^{m}\left(a_{i j}-b_{i j}\right) \leq 0 \text { for all } k, m .
$$

This condition on transition matrices is essentially equivalent to the cumulative of the bivariate distribution of $\left(y_{i}, y_{j}\right)$ being nowhere greater in society $A$ than in society $B$.

\footnotetext{
${ }^{9}$ Note that with individual risk neutrality and zero rate of pure time preference $V_{i}=y_{i}+\sum_{j=1}^{n} \mathrm{a}_{i j} y_{j}$.

${ }^{10}$ Kanbur and Stromberg (1988) is an example of a line of work which assesses the dominance properties of income distributions $p^{0}, p^{1}, \ldots, p^{t}$.

${ }^{11}$ This is also referred to as the distinction between "structural mobility" and "exchange mobility" the latter being the case where the snapshot distributions are held constant.
} 
The result in (11) gives a specific method of comparing intergenerational transition matrices. Notice that according to this criterion although $E$ dominates $I, K$ dominates $E$. Thus equality of opportunity does not necessarily lead to higher social welfare as specified here.

\section{Mobility and dynastic inequality: a characterization result}

We continue to assume a bistochastic transition matrix with the income distribution in steady state. The bistochastic assumption is restrictive but (i) it focuses attention on "pure" mobility or "exchange mobility" and (ii) as Atkinson (1981a) argues, if the income categories are quantiles then the transition matrix is by definition bistochastic. ${ }^{12}$ Notice that since the steady state of a bistochastic transition matrix has equal numbers in each category, we can normalize to the situation where there is one person in each category. We will, however, need to be careful and not give a strict cardinal interpretation to income levels $y_{\mathrm{i}}$ associated with each category.

We will further simplify the dynasty's intertemporal utility to be the expected discounted value of a given instantaneous utility function with a constant discount rate.

Then for a two period world dynasty $i$ 's lifetime expected welfare is given by

$$
V_{i}=U\left(y_{i}\right)+\gamma \sum_{j=1}^{n} a_{i j} U\left(y_{j}\right) \quad ; \quad i=1,2, \ldots, n
$$

where $\gamma$ is the discount factor (lying between 0 and 1) and $U($.$) is the instantaneous utility$ function. In this case the condition on $W($.$) in result (11) is satisfied with standard utility$ functions $U($.$) .$

However, what happens in the case of dynasties which live more than two generations? In this case the two period results of Atkinson (1981a, b), and the bivariate results of Atkinson and Bourguignon (1982), are no longer valid. Indeed, it is not exactly clear what the analogy would be for the condition on the social welfare function in the bivariate case, and how and whether even a similar class of results could be derived.

Thus the Atkinson (1981a, b) results are somewhat limited in the long lived dynasties case. In what follows we extend their analysis to the case of infinitely lived dynasties. If we define ${ }^{13}$

$$
\underset{\sim}{U}=\left(U\left(y_{1}\right), U\left(y_{2}\right), \ldots, U\left(y_{n}\right)\right)^{\prime}
$$

as the column vector of utilities of income, then we can stack the expressions in (12) to give

$$
\underset{\sim}{V}=\underset{\sim}{U}+\gamma A \underset{\sim}{U}
$$

where $V$ is the vector of dynastic expected welfares. For a $T$ generations world (14) becomes

$$
\underset{\sim}{V}=\left[I+\gamma A+\gamma^{2} A^{2}+\ldots+\gamma^{T} A^{T}\right] \underset{\sim}{U}
$$

\footnotetext{
${ }^{12}$ Key papers in the literature make further restrictive assumptions, for example the assumption that a matrix is "monotone" in other words, the rows of the matrix from a sequence of stochastic domination (Dardanoni, 1993). Dardanoni, Fiorini and Forcini (2010) argue that this assumption is borne out in reality. However, the income categories corresponding to say the deciles differ across societies, and we may also care a great deal about those.

${ }^{13}$ This approach from Kanbur and Stiglitz $(1982,1986)$ was also followed for example by Dardanoni (1993).
} 
if we let $T \rightarrow \infty$ then under standard conditions we get

$$
\underset{\sim}{V}=[I-\gamma A]^{-1} \underset{\sim}{U}
$$

Now it can be shown that

$$
\stackrel{e^{\prime}}{\sim} \underset{\sim}{V}=\frac{1}{1-\gamma} e_{\sim}^{\prime} \underset{\sim}{U} \text { where } e_{\sim}^{\prime}=(1,1, \ldots, 1)
$$

so that the sum of dynastic welfare is the same for all transition mechanisms in this class. This allows us to focus purely on the inequality of dynastic welfare. Instead of simply taking the sum of $V_{1}, V_{2}, \ldots, V_{\mathrm{n}}$, we will let social welfare be

$$
S=S\left(V_{1}, V_{2}, \ldots, V_{n}\right)
$$

where $S(\cdot, \cdot, \ldots \cdot, \cdot)$ is now a symmetric, quasi-concave function, assumptions which are meant to capture, respectively, anonymity and egalitarianism with respect to dynastic prospects.

Our object is to rank transition matrices according to the social welfare function (18). Denote $V^{A}$ and $V^{B}$ as the vector of lifetime utilities under two transition matrices A and B:

$$
\begin{aligned}
& {\underset{\sim}{V^{A}}=[I-\gamma A]^{-1}}_{\sim}^{U} \\
& {\underset{\sim}{V}}^{B}=[I-\gamma B]^{-1} \underset{\sim}{U}
\end{aligned}
$$

and let

$$
S^{A}=S\left(\underset{\sim}{V^{A}}\right) ; S^{B}=S\left(\underset{\sim}{V^{B}}\right)
$$

we can now state the basic result of this section:

Proposition $S^{A} \geq S^{B}$ for all symmetric, quasi-concave $S\left(\right.$.) for all $\left(y_{1}, y_{2}, \ldots . . y_{n}\right)$ and all $U($.) which are unique up to a positive linear transformation, if and only if there exists a bistochastic matrix $Q$ such that

$$
B=\frac{1}{\gamma}[I-Q]+A Q
$$

\section{Proof of Sufficiency}

$$
\begin{aligned}
B & =\frac{1}{\gamma}[I-Q]+A Q \\
& \Rightarrow[I-\gamma B]=[I-\gamma A] Q \\
& \Rightarrow[I-\gamma A]^{-1}=Q[I-\gamma \mathrm{B}]^{-1} \\
& \Rightarrow[I-\gamma A]^{-1} \underset{\sim}{U}=Q[I-\gamma B]^{-1} \underset{\sim}{U} \quad \text { for all }\left(y_{1}, y_{2}, \ldots . . y_{n}\right) \text { and all } U(.) \\
& \Rightarrow \sim^{A}=Q{\underset{\sim}{V}}^{B}
\end{aligned}
$$

Now if it is not the case that $V_{1}^{A} \leq V_{2}^{A} \leq \ldots \leq V_{n}^{A}$, simply permute them to give

$$
\hat{\sim}^{A}=P_{A} \hat{\sim}^{A}
$$


where the vector $\hat{V}_{\sim}^{A}$ is ordered so that $\hat{V}_{\sim 1}^{A} \leq \hat{V}_{\sim 2}^{A} \ldots \leq \hat{\sim}_{\sim \mathrm{n}}^{A}$, and $P_{A}$ is the appropriate permutation matrix. Similarly, use an appropriate permutation matrix $P_{B}$ to reorder $V_{\sim}^{B}$ to $\hat{V}^{B}$. The argument now continues as follows:

$$
\begin{aligned}
& \hat{\sim}^{A}=Q \underset{\sim}{V^{B}} \\
& \Rightarrow P_{A} \underset{\sim}{V^{A}}=P_{A} Q P_{B}^{-1} P_{B} \underset{\sim}{V^{B}} \\
& \Rightarrow{\underset{\sim}{\hat{V}}}^{A}=\left[\begin{array}{lll}
P_{A} Q & P_{B}^{-1}
\end{array}\right] \hat{\sim}^{B} \\
& \Rightarrow{\underset{\sim}{\hat{V}}}^{A}=\hat{Q} \hat{\sim}^{B}
\end{aligned}
$$

It is easy to check that the inverse of a permutation matrix is itself a permutation matrix and hence $\hat{Q}=P_{A} Q P_{B}^{-1}$ is also a bistochastic matrix. But from Dasgupta, Sen and Starrett (1973, Theorem 1),

$$
\begin{aligned}
& \hat{V}^{A}=\hat{Q} \hat{\sim}^{B}{ }^{B} \text { where } \hat{Q} \text { bistochastic } \\
=> & S\left({\underset{\sim}{V}}^{A}\right) \geq S\left(\hat{\sim}^{B}\right)
\end{aligned}
$$

for all symmetric quasi-concave functions $S($.$) . Moreover, by symmetry of the S($.) function,

$$
\begin{aligned}
& S\left({\underset{\sim}{V}}^{\mathrm{A}}\right)=S\left({\underset{\sim}{V}}^{A}\right) ; S\left(\hat{\sim}^{B}\right)=S\left(V^{B}\right) ; \text { so that } \\
& S\left({\underset{\sim}{V}}^{A}\right) \geq S\left({\underset{\sim}{V}}^{B}\right) \text { and sufficiency is proved. }
\end{aligned}
$$

\section{Proof of Necessity}

$S\left(V_{\sim}^{A}\right) \geq S\left({\underset{\sim}{V}}^{B}\right) \quad$ for all symmetric quasi-concave functions $S($.$) , all \left(y_{1}, y_{2}\right.$, $\left.\ldots, y_{n}\right)$, and all $U($.$) unique up to a positive linear$ transformation

$\Rightarrow S\left(\hat{\sim}^{\mathrm{A}}\right) \geq S\left({\underset{\sim}{V}}^{B}\right) \quad$ by symmetry of $S($.

$\Rightarrow$ there exists a bistochastic matrix $\hat{Q}$ such that

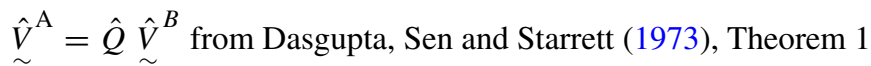

$\Rightarrow P_{A}^{-1} \hat{\sim}^{A}=P_{A}^{-1} \hat{Q} P_{B} P_{B}^{1} \hat{V}^{B}$

$\Rightarrow \underset{\sim}{V^{A}}=\left[P_{A}^{-1} \hat{Q} P_{B}\right]{\underset{\sim}{V}}^{B}$

$\Rightarrow \underset{\sim}{V^{A}}=Q \underset{\sim}{V^{B}}$

$\Rightarrow[I-\gamma A]^{-1} \underset{\sim}{U}=Q[I-\gamma B]^{-1} \underset{\sim}{U}$

$\Rightarrow[I-\gamma A]^{-1}=Q[I-\gamma B]^{-1} \quad$ Since the above equality is true for all $\left(y_{1}, y_{2}\right.$, $\ldots . ., y_{n}$ ) and all $U($.) unique up to a positive

$\Rightarrow B=\frac{1}{\gamma}[I-Q]+A Q$ linear transformation 
and proof of necessity is completed.

We thus have a characterization result on intergenerational mobility - what two patterns must look like so that we can say with confidence that dynastic inequality is lower in one than the other. ${ }^{14}$ How does the result relate to conventional views on "more mobility" or "equality of opportunity"? The next section interprets the result in light of this discourse.

\section{Interpretations}

It should be noted that the theorem of the previous section provides us with a necessary and sufficient characterization result. Within the class of bistochastic transition matrices and restricting ourselves to steady states; if we are not willing to specify the social welfare function in any greater detail than that it be symmetric and quasi-concave in dynastic expected welfares; and if we accept that lifetime utility is the present discounted sum of von-Neumann Morgenstern expected utility over an infinite horizon; then to say that social welfare is higher under transition matrix $A$ than under transition matrix $B$, is equivalent to saying that $A$ and $B$ stand in the relation

$$
B=\frac{1}{\gamma}[I-Q]+A Q
$$

where $Q$ is a bistochastic matrix. Of course, this is a very strong requirement. But under the conditions stated this is all that can be said. One route for further research may be to relax some of these conditions, but here we will restrict ourselves to relating (21) to some conventional views on "greater mobility".

Given any two bistochastic transition matrices $A$ and $B$, from (21) we simply have to check whether or not

$$
Q=[I-\gamma A]^{-1}[I-\gamma B]
$$

is a bistochastic matrix. Then, and only then, will $A$ 's welfare ranking be higher than that of $B$ for the class of social welfare functions being considered. Now using

$$
[I-\gamma A]^{-1}=\sum_{t=0}^{\infty} \gamma^{t} A^{t}
$$

it can be seen that

$$
\underset{\sim}{e^{\prime}} Q=\underset{\sim}{e^{\prime}} ; Q \underset{\sim}{e}=\underset{\sim}{e}
$$

Thus all that remains to be checked is whether each element of the matrix in (22) is nonnegative i.e. whether $q_{i j} \geq 0$.

For any two matrices, calculation of (22) and checking if the resultant matrix is nonnegative is the operational method of identifying social welfare dominance in our setting. It is the equivalent of the Lorenz curve comparison in the static case, and of the Atkinson criterion (11) in the case of measuring mobility for two period dynasties.

Let us illustrate (22) with some examples. Start with an intuitively obvious case. How does the identify matrix compare to other bistochastic matrices? Let $B=I$ in (22) and let

\footnotetext{
${ }^{14}$ Dardanoni (1993) also presents characterization results for the class of monotone matrices.
} 
$A$ be any other bistochastic matrix, noting that from the many steady state distributions for I we choose $p^{*}=\left(\frac{1}{n}, \frac{1}{n}, \frac{1}{n}, \ldots, \frac{1}{n}\right)$ to maintain comparability. Then from (22)

$$
Q=(1-\gamma)[I-\gamma A]^{-1}
$$

all of whose elements are non-negative. Thus any matrix in the bistochastic class is ranked better than the identity matrix. Moreover, letting $A=I$ in (22) we get

$$
Q=\frac{1}{1-\gamma}[I-\gamma B]
$$

some of whose off-diagonal elements will always be negative if $B$ is not itself the identity matrix. Thus $I$ is not ranked better than any other bistochastic matrix. It is in this sense that $I$ is the unique globally "worse" transition matrix in the bistochastic class if the objective is dynastic inequality.

Consider now the "equality of opportunity view", which would regard the matrix

$$
E=\frac{1}{n}\left[\begin{array}{c}
e^{\prime} \\
\sim \\
e^{\prime} \\
\sim \\
\cdot \\
\cdot \\
\cdot \\
\cdot \\
e^{\prime} \\
\sim
\end{array}\right]=\left[\begin{array}{cccc}
\frac{1}{n} & \frac{1}{n} & \ldots & \frac{1}{n} \\
\frac{1}{n} & \frac{1}{n} & \ldots & \frac{1}{n} \\
\cdot & \cdot & & \cdot \\
\cdot & \cdot & & \cdot \\
\cdot & \cdot & & \cdot \\
\cdot & \cdot & & \cdot \\
\frac{1}{n} & \frac{1}{n} & \ldots & \frac{1}{n}
\end{array}\right]
$$

as the best in the class of bistochastic transition matrices. This is of course the matrix $E$ in (5) for the bistochastic case. Let us compare this with the matrix $K$ defined in (4), where correlation between one period's income and the next period's income is the most negative it can be. First of all, let $A=E$ and $B=K$ in (22). Noting that $E^{2}=E$, it is easy to show (as in Kanbur and Mukerji (1980)) that:

$$
[I-\gamma E]^{-1}=\left[I+\frac{\gamma}{1-\gamma} E\right]
$$

so we have

$$
\begin{aligned}
\mathrm{Q} & =\left[I+\frac{\gamma}{1-\gamma} E\right][I-\gamma K] \\
& =I-\gamma K+\gamma E .
\end{aligned}
$$

In deriving the final form of (28) we have used the fact that $K E=E$. Now from (28) and the definition of $\mathrm{K}$ in (4),

$$
\begin{aligned}
& i \neq j ; \quad q_{i j}=\gamma\left(\frac{1}{n}-k_{i j}\right)=\left\{\begin{array}{l}
\gamma\left(\frac{1}{n}-1\right) \text { for } j=n-i+1 \neq i \\
\frac{\gamma}{n} \text { for } j \neq n-i+1=i
\end{array}\right. \\
& i=j ; \quad q_{i j}=1-\gamma \frac{1}{n}+\gamma \frac{1}{n}=\left\{\begin{array}{l}
1-\gamma+\gamma \frac{1}{n} \text { for } i=\frac{n+1}{2}(n \text { odd }) \\
1+\gamma \frac{1}{n} \text { for other } i
\end{array} .\right.
\end{aligned}
$$

Thus at least some elements of $Q$ (those for which $j=n-i+1 \neq i$ ) will be negative and $E$ cannot be established as unambiguously better than $K$. 
Can $K$ be established an unambiguously better than $E$ ? Letting $A=K, B=E$ in (22), and noting that

$$
[I-\gamma K]^{-1}=\frac{1}{1-\gamma^{2}}[I+\gamma K]
$$

we get

$$
\begin{aligned}
Q & =[I-\gamma K]^{-1}[I-\gamma E] \\
& =\frac{1}{1-\gamma^{2}} \mathrm{I}+\frac{\gamma}{1-\gamma^{2}} \mathrm{~K}-\frac{\gamma}{1-\gamma} E
\end{aligned}
$$

hence

$$
\begin{aligned}
& i \neq j ; q_{i j}=\frac{\gamma}{1-\gamma^{2}} k_{i j}-\frac{\gamma}{1-\gamma} \cdot \frac{1}{n}=\left\{\begin{array}{l}
\frac{\gamma}{1-\gamma^{2}}-\frac{\gamma}{1-\gamma} \cdot \frac{1}{n} \text { for } j=n-i+1 \neq i \\
\frac{\gamma}{1-\gamma} \cdot \frac{1}{n} \text { for } j \neq n-i+1 \neq i
\end{array}\right. \\
& i=j ; q_{i j}=\frac{1}{1-\gamma^{2}}+\frac{\gamma}{1-\gamma^{2}} k_{i j}-\frac{\gamma}{1-\gamma} \cdot \frac{1}{n}=\left\{\begin{array}{l}
\frac{n-\gamma(1+\gamma)}{n\left(1-\gamma^{2}\right)}+\frac{\gamma}{1-\gamma^{2}} \text { for } i=\frac{n+1}{2}(n \text { odd }) \\
\frac{n-\gamma(1+\gamma)}{n\left(1-\gamma^{2}\right)} \text { for other } i
\end{array}\right.
\end{aligned}
$$

The only dimension for which the $j \neq n-i+1$ and $j \neq i$ case is not a possibility is $n=2$. For $n=3$, the element $q_{23}$, for example, satisfies $j \neq n-i+1$ and $j \neq i$.

Thus in the $2 \times 2$ case $K$ is unambiguously ranked better than $E$. However, more generally the two cannot be ranked without specifying the social welfare function further.

In fact in the $2 \times 2$ case if we let

$$
A=\left[\begin{array}{ll}
\alpha & 1-\alpha \\
1-\alpha & \alpha
\end{array}\right] ; B=\left[\begin{array}{ll}
\beta & 1-\beta \\
1-\beta & \beta
\end{array}\right]
$$

then

$$
Q=\left[\begin{array}{ll}
q & 1-q \\
1-q & q
\end{array}\right] ; \quad q=\frac{1+\frac{1}{\gamma}-(\alpha+\beta)}{1+\frac{1}{\gamma}-2 \alpha}
$$

Clearly in this case $q$ is always non-negative and

$$
\beta \geq \alpha \Leftrightarrow q \leq 1
$$

so that if $B$ is worse than $A$ on the "diagonals view" then, and only then, is it unambiguously worse from the dynastic inequality point of view. Of course, it should be noted that in the $2 \times 2$ case the "diagonals view" and the "strong diagonals view" or "monotonicity", as Shorrocks (1978) called it coincide exactly. When presenting the incompatibility of the equality of opportunity view with monotonicity in the context of the $2 \times 2$ example. Shorrocks (1978) argued that "on balance monotonicity seems to be the less artificial restriction". The result here makes this intuition precise in the social welfare framework. ${ }^{15}$

Of course no comparable general validation of the "diagonals view" is available for $n \geq 3$.

\footnotetext{
${ }^{15}$ Amiel et al. (2013) argue that the negative correlation case is "only of theoretical interest since real world mobility data never show complete reversal between parents and children's economic positions". However, the issue is surely one of normative comparison rather than empirical regularity, in which case the negative correlation case is valid one to consider in the framework of minimizing dynastic inequality.
} 
$A$ counter example is easily presented using the result comparing $K$ and $E$ for $n=4$.

$$
K=\left[\begin{array}{llll}
0 & 0 & 0 & 1 \\
0 & 0 & 1 & 0 \\
0 & 1 & 0 & 0 \\
1 & 0 & 0 & 0
\end{array}\right] ; E=\left[\begin{array}{llll}
1 / 4 & 1 / 4 & 1 / 4 & 1 / 4 \\
1 / 4 & 1 / 4 & 1 / 4 & 1 / 4 \\
1 / 4 & 1 / 4 & 1 / 4 & 1 / 4 \\
1 / 4 & 1 / 4 & 1 / 4 & 1 / 4
\end{array}\right]
$$

On the diagonals view $K$ is superior to $E$ for $n=4$. But we already know from (32) that an unambiguous ranking on social welfare grounds is not available. More generally, for any two bistochastic matrices $A$ and $B$, let

$$
D=B-A
$$

if $B$ and $A$ stand in the relation (21), then

$$
d_{i j}=\frac{1}{\gamma}\left[\delta_{i j}-q_{i j}\right]+\sum_{\ell=1}^{n} a_{i \ell} q_{\ell j}-a_{i j}
$$

In particular,

$$
d_{i i}=\sum_{\ell=1} a_{i \ell} q_{\ell i}+\left(\frac{1}{\gamma}-a_{i i}\right)\left(1-a_{i i}\right) \geq 0
$$

Thus any two matrices which stand in the relation (21) satisfy the diagonals view. But the converse is not true: any two matrices which satisfy the diagonals view need not necessarily stand in the relation (21) to each other - the counter-example in (36) establishes that.

A general interpretation of (21) can be provided by writing it as

$$
B=\left[\frac{1}{\gamma} I\right][I-Q+A Q]
$$

$B$ can thus be seen as a matrix-weighed sum of $\frac{1}{\gamma} I$ and $A$, the weights being $Q$ and $[I-Q]$. If there exists a $Q$ such that $B$ can be written as this weighted sum, in a general sense $B$ is closer to the identity matrix than $A$. If interpreted in this wide sense, the "diagonals view" has a rationale in a social welfare function that is egalitarian with respect to dynastic prospects.

For a specific application, consider the matrices $A, B$ and $C$ given below, which are taken from Atkinson (1981a).

$$
\begin{aligned}
& A=\left[\begin{array}{llll}
0.48 & 0.42 & 0.10 & 0.00 \\
0.25 & 0.34 & 0.27 & 0.14 \\
0.19 & 0.17 & 0.37 & 0.27 \\
0.08 & 0.07 & 0.26 & 0.59
\end{array}\right] \\
& B=\left[\begin{array}{llll}
0.35 & 0.24 & 0.27 & 0.14 \\
0.26 & 0.30 & 0.27 & 0.17 \\
0.19 & 0.17 & 0.37 & 0.27 \\
0.20 & 0.29 & 0.09 & 0.42
\end{array}\right] \\
& C=\left[\begin{array}{llll}
0.44 & 0.23 & 0.19 & 0.14 \\
0.32 & 0.26 & 0.25 & 0.17 \\
0.18 & 0.36 & 0.27 & 0.19 \\
0.06 & 0.15 & 0.29 & 0.50
\end{array}\right]
\end{aligned}
$$

Matrix $C$ is a father to son, quartile to quartile, transition matrix for hourly earnings in Britain, derived from the work of Atkinson et al. (1983). Matrices $A$ and $B$ are alternative specifications of father to son occupational transition matrices which are got by adjusting 
the Goldthorpe (1980) data from its seven classes into four and enforcing bistochasticity. Atkinson (1981a) provides a fuller discussion of the procedure, but essentially matrix $A$ has been derived by shifting weight towards the diagonal and matrix $B$ by shifting weight away from the diagonal. Atkinson compares $C$ with $A$ and $C$ with $B$ using his criterion (11), and finds that $C$ and $A$ cannot be so ranked, but that "the earnings transition matrix is welfare inferior to case $B$ of the occupational status matrix".

Can the same be said of the matrices using our criterion? For $\gamma=0.5$ (an intergenerational interest rate of $100 \%$ ), we get the following $Q$ matrices:

$$
\begin{aligned}
Q_{C A}^{-1}= & {[I-\gamma C]^{-1}[I-\gamma A] } \\
= & {\left[\begin{array}{rrrr}
0.978 & -0.111 & 0.051 & 0.082 \\
0.035 & 0.953 & -0.009 & 0.021 \\
-0.002 & 0.095 & 0.947 & -0.040 \\
-0.011 & 0.063 & 0.011 & 0.938
\end{array}\right] } \\
Q_{B C}^{-1}= & {[I-\gamma B]^{-1}[I-\gamma C] } \\
= & {\left[\begin{array}{rrrr}
0.948 & -0.001 & 0.049 & 0.003 \\
-0.034 & 0.016 & 0.016 & 0.002 \\
0.009 & -0.101 & 0.050 & -0.042 \\
0.076 & 0.086 & -0.115 & 0.953
\end{array}\right] }
\end{aligned}
$$

As in the case of Atkinson's criterion, $C$ cannot be ranked as better than $A$. However, what is interesting is that $B$ cannot be ranked as better than $C$, in contrast to the Atkinson finding. Thus while it should be clear that there are a number of empirical problems in comparing earnings transition matrices with occupational ones, our calculations nevertheless indicate that a stress on dynastic inequality could alter rankings which emphasize intertemporal nonseparability.

\section{Conclusion}

The normative riposte to concerns about sharply rising inequality of income and wealth, that it is intergenerational mobility and equality of opportunity which really matter, need to be assessed with respect to specific social welfare functions which make explicit the value judgments underlying the claim. An earlier literature, starting with the work of Shorrocks (1978), Atkinson (1981a and 1981b), Atkinson and Bourguignon (1982) attempted to do just this, and developed criteria for assessment of alternative patterns of intergenerational mobility. Our contribution is in this vein, focusing on the implications of intergenerational mobility for inequality among infinitely lived dynasties.

Restricting attention to bistochastic transition matrices in order to hold constant the steady state snapshot income distribution, we have developed an explicit partial ordering which ranks matrices on the criterion of dynastic inequality. A general interpretation of our result is that when comparing two matrices, if one matrix is "further away" from the identity matrix then it will lead to lower dynastic inequality. More specifically, the result presents a computational procedure to check if one matrix dominates another on dynastic inequality. We can also assess "equality of opportunity", defined as identical prospects irrespective of starting position, or identical rows of a transition matrix. We find that this is not necessarily the mobility pattern which minimizes dynastic inequality, which requires instead a degree of negative correlation between intergenerational outcomes. 
Much remains to be done. At the theoretical level, we can sharpen our ordering by restricting the class of transition matrices, for example to those which satisfy stochastic monotonicity as suggested by Dardanoni, Fiorini and Forcini (2010). The criterion we have developed can be applied to intergenerational transition matrices which are now being estimated with newly available data. The earlier literature on normative measurement of mobility needs to be revisited and extended to address the revived concerns on inequality and mobility. Most importantly, the normative implications of the current inequality of income for intergenerational inequality need to be assessed again with respect to alternative sets of social welfare value judgments, perhaps bolstered by empirical investigation of peoples' views on mobility and inequality in its different dimensions, as developed by Amiel et al. (2013). Specifically, if "equality of opportunity" as captured identical rows of a transition matrix (independence of future prospects from current income or wealth), does indeed have normative significance as the best transition matrix, how do we reconcile this with the outcome that it does not necessarily minimize dynastic inequality?

Acknowledgments We first started thinking about this issue in Oxford more than thirty years ago (see Kanbur and Stiglitz $(1982,1986)$. We have returned to that line of enquiry and are reviving our earlier work given the recent tendency to counter rising inequality concerns with the argument that what matters is not inequality but mobility.

\section{References}

Aaberge, R., Mogstad, M., Peragine, V.: Measuring long-term inequality of opportunity. J. Public Econ. 95(3), 193-204 (2011)

Amiel, Y., Bernasconi, M., Cowell, F., Dardanoni, V.: Do we value mobility? London School of Economics STCERD Discussion Paper, Number PEP 17, http://sticerd.lse.ac.uk/dps/pep/pep17.pdf (2013)

Arneson, R.: Four conceptions of equal opportunity, http://bfi.uchicago.edu/sites/default/files/research/ Arneson.pdf (2014)

Atkinson, A.B.: On intergenerational income mobility in Britain. J. Post Keynesian Econ. 3, 194-218 (1981a)

Atkinson, A.B.: The measurement of economic mobility. In: Eigjelshoven, P.J., van Gemerden, L.J. (eds.) Inkomensverdeling en openbare financien Essays in Honour of Jan Pen, pp. 9-24. Het Spectrum, Utrecht (1981b). Also published in Social Justice and Public Policy (ed. A.B. Atkinson), Wheatsheaf Books Ltd, 1983, pp. 61-75.

Atkinson, A.B., Bourguignon, F.: The comparison of multi-dimensional distributions of economic status. Rev. Econ. Stud. 49, 183-201 (1982). Also published in Social Justice and Public Policy (ed.A.B. Atkinson), Wheatsheaf Books Ltd, 1983, pp. 37-59.

Atkinson, A.B., Maynard, A.K., Trinder, C.G.: Parents and Children: Incomes in Two Generations. Heinemann Educational Books, London (1983)

Chakravarty, S.R., Dutta, B., Weymark, J.A.: Ethical indices of income mobility. Soc. Choice Welf. 2, 1-21 (1985)

Corak, M.: Income inequality, equality of opportunity and intergenerational mobility. J. Econ. Perspect. 27(3, Summer), 79-102 (2013)

Dardanoni, V.: Measuring social mobility. J. Econ. Theory 61, 372-394 (1993)

Dardanoni, V., Fiorini, M., Forcina, A.: Stochastic monotonicity in intergenerational mobility tables. J. Appl. Econom. 27(1), 85-107 (2010)

Dasgupta, P., Sen, A.K., Starrett, D.: Notes on the measurement of inequality. J. Econ. Theory 6(2), 180-187 (1973)

Dworkin, R.: What is equality? Part 2: equality of resources. Philos. Public Affairs 10(4), 283-345 (1981)

Fields, G.S., Ok, E.A.: The measurement of income mobility: an introduction to the literature. In: Silber, J. (ed.) Handbook on Income Inequality Measurement, pp. 557-596. Kluwer Academic Publishers, Norwell (1999)

Friedman, M.: Capitalism and Freedom. University of Chicago Press (1962) 
Goldthorpe, J.H.: Social Mobility and Class Structure in Modern Britain. Oxford University Press, London (1980)

Kanbur, R., Mukerji, V.: Probabilistic equity and equality of opportunity in a stochastic framework. Arthaniti XX, 1-9 (1980)

Kanbur, R., Stiglitz, J.E.: Mobility and inequality: a utilitarian analysis. Economic Theory Discussion Paper No. 57, Department of Applied Economics, University of Cambridge (1982)

Kanbur, R., Stiglitz, J.E.: Intergenerational mobility and dynastic inequality. Woodrow Wilson School Discussion Paper in Economics, Number 111, Princeton University (1986)

Kanbur, R., Stromberg, J.O.: Income transitions and income distribution dominance. J. Econ. Theory 45(2), 408-417 (1988)

Kanbur, R., Wagstaff, A.: How useful is inequality of opportunity as a policy construct? In: K. Basu and J. E. Stiglitz (eds.) Chap. 4 in Inequality and Growth: Patterns and Policy, Volume I: Concepts and Analysis. Palgrave Macmillan (2016)

Markandya, A.: The welfare measurement of changes in economic mobility. Economica 51, 457-71 (1984)

Piketty, T.: Capital in the Twenty-First Century. Harvard University Press (2014)

Roemer, J.E.: Equality of Opportunity. Harvard University Press (1998)

Roemer, J.E.: Equal opportunity and intergenerational mobility: going beyond intergenerational income transition matrices. In: Corak, M. (ed.) Chap. 3 in Generational Income Mobility in North America and Europe. Cambridge University Press (2004)

Shorrocks, A.F.: The measurement of mobility. Econometrica 46(5), 1013-1024 (1978)

Stiglitz, J.E.: The Price of Inequality: How Today's Divided Society Endangers Our Future. W.W. Norton and Company (2013)

Stokey, N.: Shirtsleeves to shirtsleeves: the economics of social mobility. In: Schwartz, N.L., Jacobs, D., Kalai, E. (eds.) Frontiers of Research in Economic Theory: the Nancy L. Schwartz Memorial Lectures 1983-1997, pp. 210-241. Econometric Society Monographs, Cambridge (1998) 